\title{
Educação a Distância - tradução, adaptação e validação da escala de motivação EMITICE
}

\author{
Patricia Jantsch Fiuza \\ Jorge Castellá Sarriera \\ Lívia Maria Bedin
}

\begin{abstract}
Resumo
A motivação interfere na forma das pessoas interagirem e nas relações com outros, destaca-se neste estudo a motivação no uso de TIC na educação. Este artigo relata a tradução, adaptação e validação da Escala EMITICE, baseada na Teoria da Autodeterminação (TAD). Realizaramse duas fases: (1) tradução, adaptação e teste piloto ( $N=91),(2)$ teste de campo e validação $(\mathrm{N}=466)$ em alunos brasileiros de cursos a distância, idades entre 18 a 61 anos $(M=34,48$; $D P=9,56), 171$ homens $(36,7 \%)$ e 295 mulheres (63,3\%). Utilizaram-se juízes bilíngues, síntese das traduções, comitê de especialistas e backtranslation. Correlação de Pearson, análise fatorial confirmatória e consistência interna demonstram que a EMITICE é válida e fidedigna. A AFC apresentou adequação ao modelo e boa consistência interna na amostra $(\alpha=0,84)$. Os resultados reforçam as qualidades psicométricas, indicando aplicabilidade em estudos sobre motivação em relação às TIC, constituindo-se num instrumento teórica e empiricamente embasado, útil à pesquisa científica.
\end{abstract}

Palavras-chave: Avaliação, motivação, comunicação e tecnologia.

\section{Distance education:}

\section{translating, adaptating and validating the motivation emitice scale}

\begin{abstract}
Motivation interferes in the way people interact and relate with others. In this study we focus on the motivation in the use of ICT in education. We explore translation, adaptation and validation of EMITICE Scale based on Self-Determination Theory (SDT). The study has been made in two phases: (1) translation, adaptation and pilot testing ( $N=91),(2)$ field test and validation $(N=466)$ with distance learning Brazilian students, aged 18 to $61(\mathrm{M}=34.48, \mathrm{SD}=9.56), 171$ men $(36.7 \%)$. This study used bilingual referees, translations synthesis, expert committee and backtranslation. Pearson correlation, confirmatory factor analysis and internal consistency show that EMITICE is valid and reliable. The CFA shows adequacy model and good internal consistency $(\alpha=0.84)$. The results reinforce psychometric qualities of scale indicating its applicability in studies of motivation in the use of ICT thus becoming an instrument theoretically and empirically grounded useful for scientific research.
\end{abstract}

Keywords: Evaluation, motivation, communication and technology.

\section{Educación a distancia:}

\section{traducción, adaptación y validación de la escala de motivación emitice}

\section{Resumen}

La motivación interfiere en la forma como las personas interactúan y en las relaciones con los demás, se destaca en este estudio la motivación en el uso de TIC en la educación. Este artículo relata la traducción, adaptación y validación de la Escala EMITICE, basada en la Teoría de la Autodeterminación (TAD). Se realizaron dos fases: (1) traducción, adaptación y test piloto ( $N=91)$, (2) test de campo y validación ( $N=466)$ en alumnos brasileños de cursos a distancia, edades entre 18 a 61 años ( $M=34,48$; $D S=9,56), 171$ hombres $(36,7 \%)$ y 295 mujeres $(63,3 \%)$. Se utilizaron jueces bilingües, síntesis de las traducciones, comité de especialistas y backtranslation. Correlación de Pearson, análisis factorial confirmatoria y consistencia interna demuestran que la EMITICE es válida y fidedigna. La AFC presentó adecuación al modelo y buena consistencia interna en la muestra $(\alpha=0,84)$. Los resultados refuerzan las cualidades psicométricas indicando aplicabilidad en estudios sobre motivación en relación a las TIC constituyéndose un instrumento teórica y empíricamente fundamentado, útil a la investigación científica.

Palabras clave: Evaluación, motivación, comunicación y tecnología. 


\section{Introdução}

A área da avaliação psicológica vem se modificando nas últimas décadas. Atualmente, no Brasil, encontram-se vários estudos na área de avaliação, porém insuficientes para atender as necessidades da psicologia brasileira, indicando a necessidade de mais pesquisas em relação aos instrumentos psicológicos (Noronha, Freitas, Sartori, \& Ottati, 2002). A partir desse contexto, o Conselho Federal de Psicologia (CFP) vem promovendo debates e discussões acerca da Resolução 25/2001, que regulamentou normas para os manuais dos testes a serem atualizados, e da Resolução 002/2003, que propôs um sistema de avaliação dos testes psicológicos.

Dentre os procedimentos de diagnóstico e prognóstico na psicologia, os testes são uma das manifestações ou técnicas da área (Pasquali, 2001). De acordo com Anastasi e Urbina (2003), o valor diagnóstico ou preditivo de um teste depende do grau em que ele serve como indicador de uma área relativamente ampla e significativa de comportamento, e o fato desse comportamento poder servir como um índice efetivo de outros comportamentos é algo que só pode ser estabelecido por provas empíricas.

A utilização de instrumentos de avaliação psicológica permite, também, maior qualidade nas pesquisas do profissional da psicologia e consequentemente nas outras áreas científicas que precisem das contribuições dessa ciência. A avaliação psicológica deve ser um processo integrado, utilizando técnicas apropriadas para diagnosticar cada situação visando a alguma intervenção (Pasquali, 2001). Vale destacar a visão de Primi, Muniz e Nunes (2009) de que a avaliação envolve desde conhecimentos teóricos no entendimento do funcionamento psicológico até a compreensão e previsão do comportamento de pessoas e grupos, ou seja, é uma importante competência do profissional de psicologia.

A proposta deste artigo é expor parte da pesquisa referente à tradução, adaptação e validação da Escala EMITICE - Escala de Avaliação de Fatores de Motivação com Relação à Integração das Tecnologias de Informação e Comunicação ao Ensino, com base na escala canadense originalmente aplicada na Universidade de Montreal (Karsenti, 2008) denominada EMITICE - Echelle de motivation lor de l'intégration des technologies de l'information et des communications dans l'enseignement (Karsenti, 2008). A pesquisa surgiu a partir da relevância apresentada pela variável motivação e pelos recursos tecnológicos de informação e comunicação para as pessoas em várias situações, em especial no atual cenário da educação brasileira, onde a modalidade a distância tem crescido muito.

Os fatores motivacionais podem ser determinantes na forma das pessoas interagirem e se colocarem em suas relações com os outros e com aspectos da vida em si. A compreensão atual da motivação direciona para aspectos intrínsecos dos indivíduos. Segundo Ryan e Deci (2000), estar motivado significa ser movido para fazer alguma coisa. A pessoa que não sente nenhum impulso ou inspiração para agir é, portanto, caracterizada como desmotivada, enquanto alguém que está energizado ou ativado em direção a um fim é considerado motivado. As pessoas têm não só diferentes quantidades, mas também diferentes tipos de motivação. Dessa forma, variam não apenas em nível de motivação (ou seja, o quanto de motivação), mas também na orientação de qual motivação (ou seja, qual tipo de motivação).

Ryan e Deci (2000) desenvolveram estudos sobre a motivação e chegaram à Teoria da Autodeterminação (TAD), que é uma macroteoria da motivação composta de quatro subteorias: a teoria da avaliação cognitiva, a teoria da integração organísmica, a teoria das necessidades básicas e a teoria das orientações de causalidade. Assim, a teoria da autodeterminação distingue entre diferentes tipos de motivação com base nas razões ou objetivos diferentes que dão origem a uma ação. A distinção mais básica é entre a motivação intrínseca, que se refere a fazer algo porque é inerentemente interessante ou agradável, e motivação extrínseca, que se refere a fazer algo, pois leva a um resultado esperado. Mais de três décadas de pesquisas mostraram que a qualidade da experiência e desempenho pode ser muito diferente quando se está se comportando por razões intrínsecas ou extrínsecas (Ryan \& Deci, 2000).

Ainda para esses autores, na literatura clássica, a motivação extrínseca tem sido tipicamente caracterizada como uma pálida e empobrecida forma (mesmo que poderosa) de motivação, que contrasta com a motivação intrínseca. Na subteoria da integração organísmica, os autores propõem um continuum de autodeterminação que existiria a partir de diferentes tipos de motivação com início na desmotivação, seguidos pela regulação externa, regulação introjetada, regulação identificada e regulação integrada, terminando com a motivação intrínseca (Ryan \& Deci, 2000). Na figura 1, é apresentada a síntese da taxonomia da motivação humana para a TAD.

Motivação intrínseca representa envolvimento em uma atividade por si mesma. Já a motivação extrínseca foi diferenciada nos tipos de regulação já citados, que variam em seu grau de autonomia relativa. Com essa extensão, o principal foco mudou para a motivação autônoma em relação à motivação controlada. Motivação autônoma envolve a experiência da vontade e escolha, enquanto que a motivação controlada envolve a experiência de ser pressionado ou coagido (Vansteenkiste, Lens, \& Deci, 2006). Comportamentos são autônomos na medida em que pessoas experimentam um verdadeiro sentido de vontade, escolha e ato por causa da importância pessoal do comportamento. Em contraste, comportamentos são controlados na medida em que as pessoas se sentem pressionados para realizá-los, tanto por forças externas ou intrapsíquicas (Williams, Gagné, Ryan, \& Deci, 2002).

A subteoria das necessidades básicas na TAD se concentra principalmente em necessidades psicológicas, ou seja, as necessidades inatas de competência, autonomia e relacionamento e essas necessidades básicas devem ser atendidas pelos comportamentos intrinsecamente motivados (Ryan \& Deci, 2000). A satisfação das três necessidades inatas é essencial para um ótimo desenvolvimento e saúde psicológica dos indivíduos (Guimarães \& Boruchovitch, 2004). 


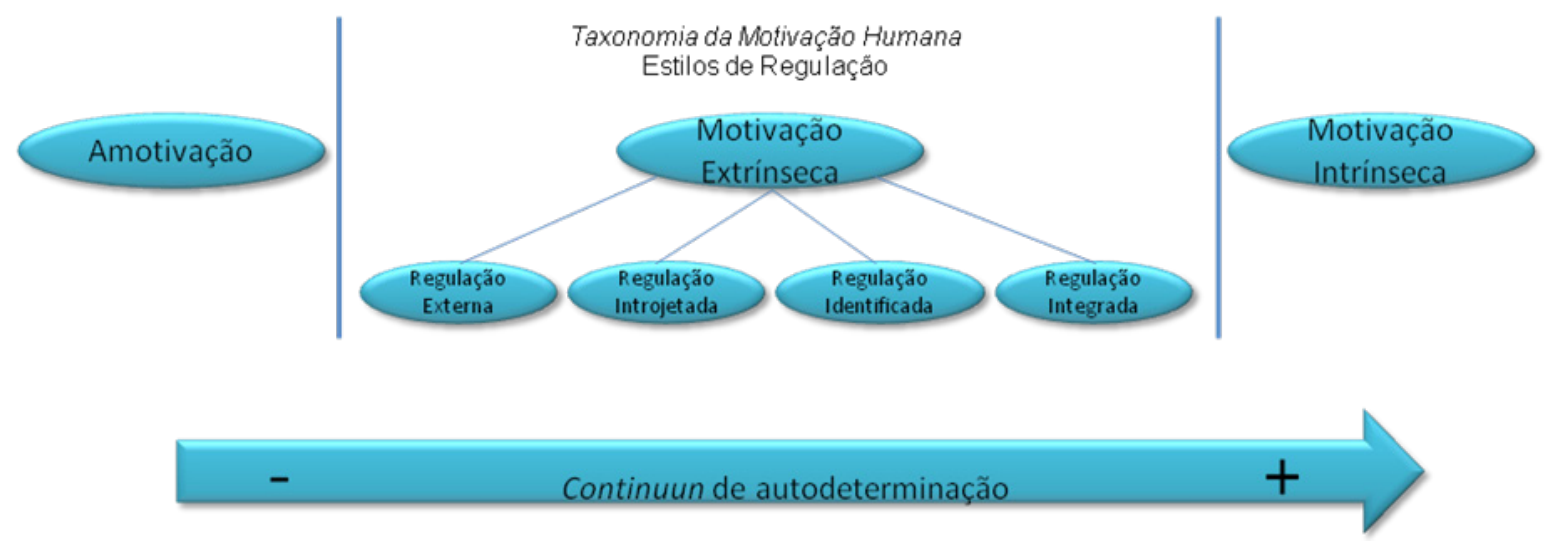

Figura 1. Taxonomia da Motivação Humana na Teoria da Autodeterminação

Nota. Adaptado de Ryan e Deci (2000)

A autonomia é vinculada ao desejo, a vontade do organismo de organizar as experiências e o próprio comportamento, é a vontade e a autorregulação integrada à autodireção, ou à autodeterminação. Já a competência é a capacidade do organismo de interagir satisfatoriamente com o seu meio. Assim, a experiência de dominar uma tarefa desafiadora e o aumento da competência dela resultante trazem emoções positivas denominadas sentimento de eficácia. Segundo Guimarães e Boruchovitch (2004, p. 146), "os eventos sócio-contextuais que fortalecem a percepção de competência no decorrer de uma ação, por exemplo, o feedback positivo em situações de desafio de nível ótimo, aumentam a ocorrência da motivação intrínseca". E a terceira necessidade básica, relacionamento ou estabelecer vínculos, é considerada menos central na motivação intrínseca do indivíduo, vista apenas como "pano de fundo", ou seja, como segurança para um desenvolvimento saudável (Guimarães \& Boruchovitch, 2004).

A partir da relevância do tema exposto, a intenção deste estudo é contribuir para futuras pesquisas brasileiras nas áreas da Psicologia e da Educação, em especial à Educação a Distância. Busca-se fornecer uma medida objetiva dos comportamentos nos quais as pessoas são direcionadas pela motivação, principalmente em relação ao uso dos recursos tecnológicos.

\section{Objetivo}

Este estudo teve como objetivo traduzir, adaptar e validar a EMITICE - Escala de Avaliação de Fatores de Motivação com Relação à Integração das Tecnologias de Informação e Comunicação ao Ensino - para a realidade brasileira.

\section{Método}

Fase 1: Tradução da escala EMITICE por especialistas e adaptação da escala à realidade cultural brasileira e teste piloto

Beaton, Bombardier, Guillemin e Ferraz (2000) apresentam um esquema com seis estágios que compõem o processo de tradução e adaptação transcultural, que são: 1) Tradução (dois tradutores independentes, um leigo e um expert); 2) Síntese (onde se soma mais um tradutor para resolver discrepâncias); 3) Tradução Reversa (dois tradutores); 4) Revisão por Comitê de Especialistas; 5) Estudo Piloto; e 6) Submissão da versão final aos criadores ou responsáveis pela adaptação do instrumento.

No caso da EMITICE, a tradução por especialistas (adaptação dos passos do método de Beaton e cols., 2000) compôs-se de cinco etapas: (1) tradução para o português (brasileiro) por três juízes bilíngues independentes; (2) síntese, por comparação, das três versões iniciais; (3) revisão da síntese por um comitê de especialistas, para resolver divergências, buscando, entre original e versão, equivalência semântica, idiomática, conceitual e vivencial de cada item do instrumento; (4) submissão dos itens da Escala ao procedimento de backtranslation (tradução reversa do instrumento para as línguas inglesa e francesa), o que foi realizado por outros três juízes; e, para finalizar, o (5) Estudo Piloto.

\section{Procedimentos}

Para o estudo piloto, foi realizada uma versão preliminar da escala traduzida, que foi testada com um pequeno grupo de sujeitos para possibilitar a verificação da fidedignidade dos constructos e a validade da escala com a amostra. 
O piloto foi realizado em amostra de 91 universitários de duas instituições de ensino superior, uma particular e outra pública, da região sul do Brasil (SC e RS), que ofereciam cursos de graduação na modalidade de EaD para alunos de todo o Brasil.

Os dados foram coletados através de um formulário de pesquisa com as questões do instrumento, disponibilizadas em um site na internet. Todos participantes concordaram em participar, aceitando o Termo de Consentimento Livre e Esclarecido, e foram consideradas todas as questões éticas de pesquisa com seres humanos neste estudo, que foi submetido e aprovado pelo comitê de ética. Os resultados encontrados no teste piloto indicaram pequenas alterações de redação com adaptações a termos mais compreensíveis em alguns itens da escala para melhor adequação cultural da mesma.

\section{FASE 2: Teste de campo e validação da Escala EMITICE}

\section{Participantes}

A amostra final deste estudo foi constituída por 466 sujeitos, com idade média de 34,48 (SD = 9,56), de várias instituições de ensino superior da região sul do Brasil (SC, RS e PR) que ofertam cursos de graduação na modalidade a distância para alunos de todo o Brasil. Como pode se observar na Tabela 1, a maior parte está cursando graduação.

Tabela 1. Frequências e percentuais da amostra por gênero e escolaridade

\begin{tabular}{lll}
\hline & & N total $(\%)$ \\
\hline Gênero & Feminino & $295(63,3)$ \\
\hline Escolaridade* $^{*}$ & 1 & $10(2,1)$ \\
& 2 & $268(57,5)$ \\
& 3 & $62(13,3)$ \\
& 4 & $41(8,8)$ \\
& 5 & $73(15,7)$ \\
& 6 & $10(2,1)$ \\
& 7 & $1(0,2)$ \\
& 8 & $1(0,2)$ \\
\hline
\end{tabular}

*1 = Ensino Médio, 2 = Cursando Graduação, 3 = Graduado, 4 = Cursando Pós-graduação, 5 = Especialização, 6 = Mestrado, 7 = Doutorado, $8=$ Outros

\section{Instrumentos}

Para a realização deste estudo, foram utilizados o Questionário Sociodemográfico (dados demográficos, socioeconômicos e de estudo) e a Escala EMITICE. O questionário sóciodemográfico serviu para caracterizar a amostra em termos de perfil por gênero, idade, escolaridade e tipo de instituição que frequentava. A EMITICE passou por um processo que envolveu a tradução, adaptação e validação por meio de análises entre juízes e estatísticas. O instrumento foi gentilmente disponibilizado pela equipe de pesquisadores da Universidade de Montreal. Trata-se de uma escala desenvolvida originariamente por Vallerand, Blais, Brière e Pelletier em 1989 e que se baseia na teoria motivacional de Deci e Ryan. Pesquisadores da Universidade de Montreal utilizaram a escala em estudos que revelaram bons níveis de fidelidade, com consistência interna relativamente elevada $(0,74$ a 0,91$)$ e validade também adequada, constatada por meio de uma análise fatorial efetuada sobre o conjunto dos dados recolhidos (Karsenti, 2008). A escala contém 21 itens que correspondem a cinco dimensões: desmotivação, controle externo, controle interno, controle por identificação e motivação intrínseca, sendo respondida por uma medida de 7 pontos tipo Likert, variando de "1 - Não corresponde absolutamente" até "7 - Corresponde absolutamente".

\section{Resultados}

Para verificar as propriedades psicométricas da EMITICE, foi realizada a correlação entre os itens da escala. Posteriormente, realizou-se análise fatorial confirmatória, com método de estimação por máxima verossimilhança e análise de consistência interna para avaliar a fidedignidade e a validade da escala.

\section{Correlações entre os itens}

Foi realizada análise para verificar as correlações entre os 21 itens da EMITICE, conforme pode ser visto na Tabela 2. As correlações de Pearson mostram que existem correlações positivas e significativas entre a maior parte dos itens. Os itens 2, 4, 7 e 20 apresentaram as correlações mais baixas com os demais itens, sendo algumas correlações não significativas. Os itens 5, 9, 10, 14, 15 e 19 apresentaram correlações moderadas entre os demais itens, com poucas correlações não significativas. Os itens 1, 3, 6, 8, 11, 12, 13, $16,17,18$ e 21 foram os que apresentaram maiores correlações com o maior número de itens. O objetivo de realizar a correlação entre os itens e não a correlação item-total busca verificar as relações entre os itens de um mesmo fator latente, assim como a ausência de correlação ou baixa correlação entre os itens de um mesmo fator com os demais fatores, assumindo que são interdependentes. 


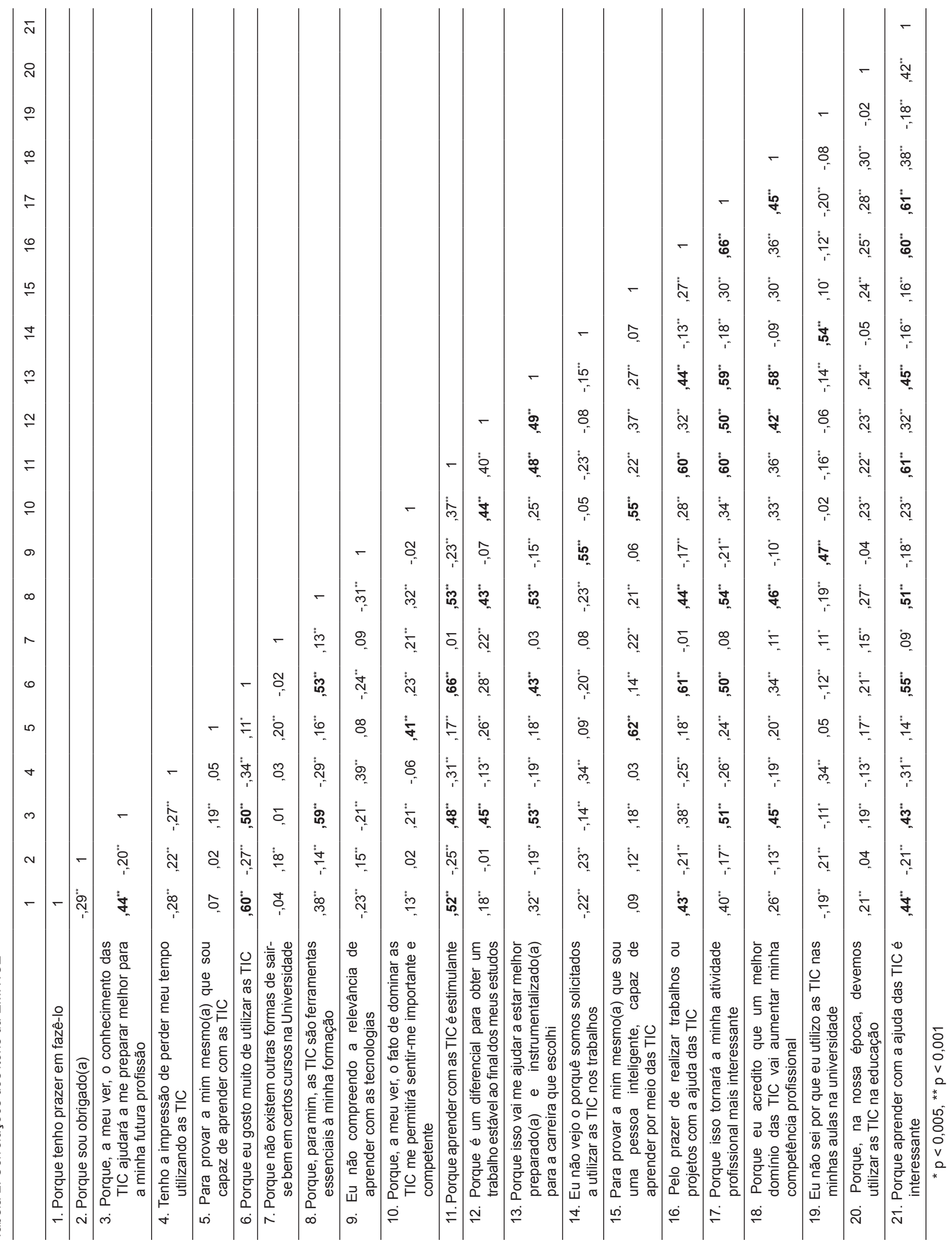




\section{Análise Fatorial Confirmatória}

Foi realizada análise fatorial confirmatória (AFC) com os 21 itens da EMITICE, considerando-se cinco fatores. Os pressupostos para realização da análise foram atendidos, sendo verificados os pressupostos de normalidade e homoscedasticidade das variáveis. A análise fatorial confirmatória se destina a verificar o comportamento de variáveis observadas e latentes a partir de um modelo teórico estabelecido a priori a partir da literatura (Batista-Foguet \& Coenders, 2000; Byrne, 2010; Hair, Anderson, Tatham, \& Black, 2005).

Para o modelo ser considerado satisfatório, foram consideradas as indicações de Batista-Foquet e Coenders (2000), que, além do Qui-quadrado, apontam o uso do Índice de Comparação do Ajuste (Comparative Fit Index de Bentler
- CFI) e a análise dos resíduos através do Erro Quadrático Médio de Aproximação (Root Mean Square Error of Approximation - RMSEA) e seus intervalos de confiança. A AFC apresentou bons índices de ajuste, com $\chi 2(152)=381,5$, $p<0,001$, e índices de comparação do ajuste do modelo empírico da amostra com o modelo teórico de $N F I=0,908$, $T L I=0,927$ e $C F I=0,942$. Os resíduos também foram considerados aceitáveis, apresentando RMSEA de 0,057, com intervalo de confiança de 0,050 a 0,064.

A Figura 2 mostra a representação gráfica do modelo de AFC da EMITICE, considerando seus cinco fatores latentes determinados pela teoria. Também são apresentadas as covariâncias entre os erros apontadas pelos índices de modificação, referindo principalmente correlações entre erros de controle interno e motivação intrínseca.

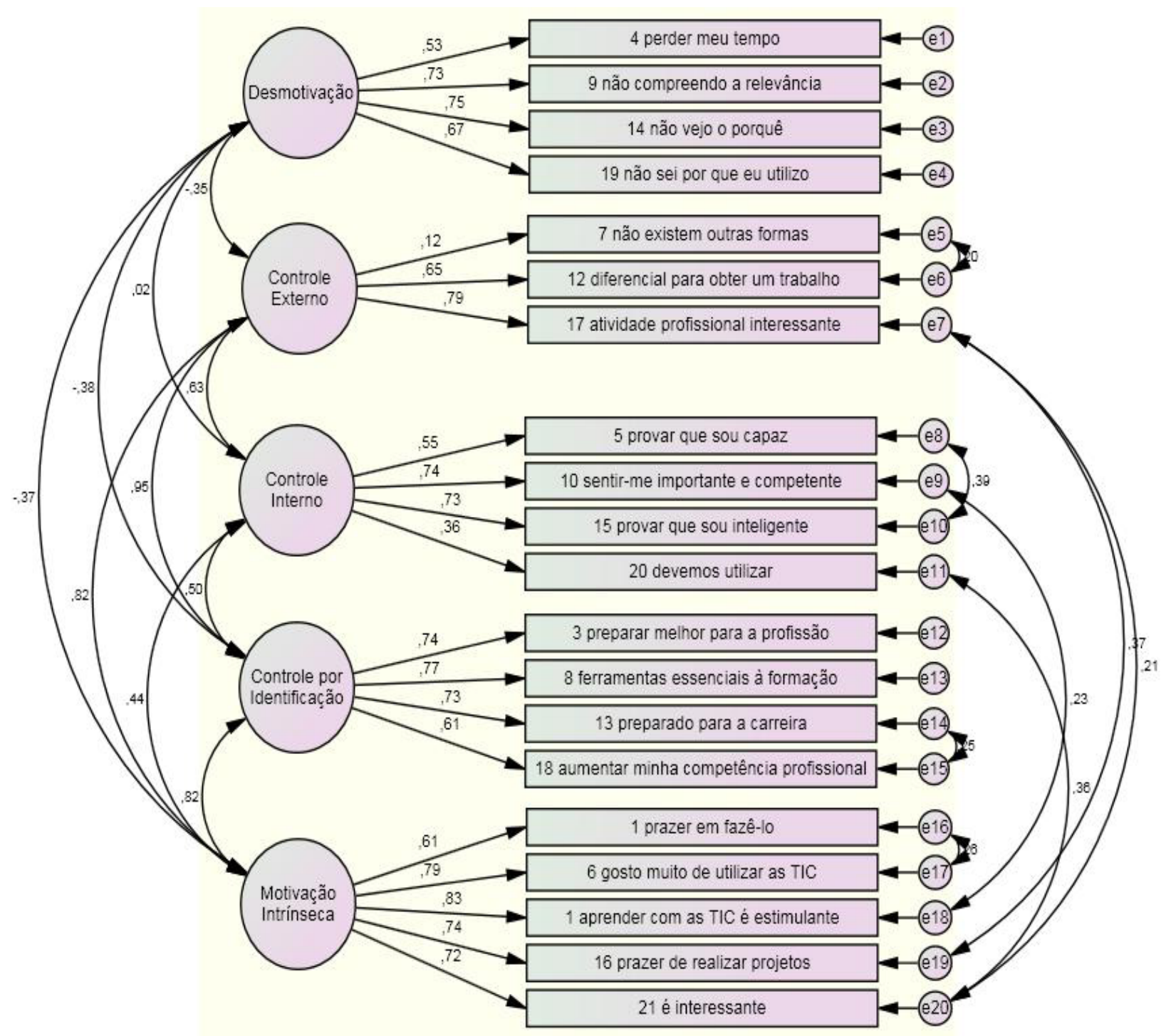

Figura 2. Análise Fatorial Confirmatória da EMITICE com cargas padronizadas 
Foram considerados apenas os itens que apresentaram cargas fatoriais positivas e estatisticamente significativas $(p<0,001)$. Optou-se por retirar o item 2 "Porque sou obrigado(a)" pois apresentou carga fatorial negativa, o que é apontado por Byrne (2010) como uma violação dos critérios de viabilidade de estimativas de parâmetros de uma AFC. Além disso, esse item também não apresentou carga fatorial significativa ao fator latente Controle Externo $(p>0,05)$. A Tabela 3 apresenta as cargas fatoriais dos itens da EMITICE com seus respectivos fatores latentes.
A consistência interna da EMITICE para a amostra deste estudo foi de Alpha de Cronbach de 0,84. Com relação aos cinco fatores, o primeiro fator, denominado Desmotivação, apresentou $\alpha=0,76$, o segundo, denominado Controle Externo, obteve uma consistência interna de alpha de 0,51. O terceiro, denominado Controle Interno, apresentou alpha de 0,71 , o quarto fator, denominado Controle por Identificação, apresentou alpha de 0,81 , e o quinto fator, denominado Motivação Intrínseca, obteve alpha de 0,87.

Tabela 3. Cargas fatoriais padronizadas da escala EMITICE para amostra

\begin{tabular}{|c|c|c|c|}
\hline & & & $\begin{array}{l}\text { Cargas } \\
\text { Fatoriais }\end{array}$ \\
\hline Tenho a impressão de perder meu tempo utilizando as TIC & $<---$ & Desmotivação &, 525 \\
\hline Eu não compreendo a relevância de aprender com as tecnologias & $<---$ & Desmotivação & ,730 \\
\hline $\begin{array}{l}\text { Eu não vejo o porquê somos solicitados a utilizar as TIC nos } \\
\text { trabalhos }\end{array}$ & $<---$ & Desmotivação & ,748 \\
\hline $\begin{array}{l}\text { Eu não sei por que eu utilizo as TIC nas minhas aulas na } \\
\text { universidade }\end{array}$ & $<---$ & Desmotivação & ,674 \\
\hline $\begin{array}{l}\text { Porque não existem outras formas de sair-se bem em certos } \\
\text { cursos na Universidade }\end{array}$ & $<---$ & Controle Externo & ,118 \\
\hline $\begin{array}{l}\text { Porque é um diferencial para obter um trabalho estável ao final } \\
\text { dos meus estudos }\end{array}$ & $<---$ & Controle Externo & ,645 \\
\hline $\begin{array}{l}\text { Porque isso tornará a minha atividade profissional mais } \\
\text { interessante }\end{array}$ & $<---$ & Controle Externo & ,794 \\
\hline $\begin{array}{l}\text { Para provar a mim mesmo(a) que sou capaz de aprender com as } \\
\text { TIC }\end{array}$ & $<---$ & Controle Interno &, 554 \\
\hline $\begin{array}{l}\text { Porque, a meu ver, o fato de dominar as TIC me permitirá sentir- } \\
\text { me importante e competente }\end{array}$ & $<---$ & Controle Interno & ,737 \\
\hline $\begin{array}{l}\text { Para provar a mim mesmo(a) que sou uma pessoa inteligente, } \\
\text { capaz de aprender por meio das TIC }\end{array}$ & $<---$ & Controle Interno & ,728 \\
\hline Porque, na nossa época, devemos utilizar as TIC na educação & $<--$ & Controle Interno &, 360 \\
\hline $\begin{array}{l}\text { Porque, a meu ver, o conhecimento das TIC ajudará a me } \\
\text { preparar melhor para a minha futura profissão }\end{array}$ & $<---$ & $\begin{array}{l}\text { Controle por } \\
\text { Identificação }\end{array}$ & ,737 \\
\hline $\begin{array}{l}\text { Porque, para mim, as TIC são ferramentas essenciais à minha } \\
\text { formação }\end{array}$ & $<---$ & $\begin{array}{l}\text { Controle por } \\
\text { Identificação }\end{array}$ & ,768 \\
\hline $\begin{array}{l}\text { Porque isso vai me ajudar a estar melhor preparado(a) e } \\
\text { instrumentalizado(a) para a carreira que escolhi }\end{array}$ & $<---$ & $\begin{array}{l}\text { Controle por } \\
\text { Identificação }\end{array}$ & ,731 \\
\hline $\begin{array}{l}\text { Porque eu acredito que um melhor domínio das TIC vai aumentar } \\
\text { minha competência profissional }\end{array}$ & $<---$ & $\begin{array}{l}\text { Controle por } \\
\text { Identificação }\end{array}$ & ,607 \\
\hline Porque tenho prazer em fazê-lo & $<--$ & Motivação Intrínseca &, 606 \\
\hline Porque eu gosto muito de utilizar as TIC & $<--$ & Motivação Intrínseca &, 788 \\
\hline Porque aprender com as TIC é estimulante & $<--$ & Motivação Intrínseca &, 829 \\
\hline Pelo prazer de realizar trabalhos ou projetos com a ajuda das TIC & $<--$ & Motivação Intrínseca &, 743 \\
\hline Porque aprender com a ajuda das TIC é interessante & $<---$ & Motivação Intrínseca & ,721 \\
\hline
\end{tabular}




\section{Discussão}

O processo de tradução e adaptação do instrumento EMITICE seguiu os passos descritos na literatura e permitiu a análise psicométrica da escala em sua versão para o português brasileiro (Geisinger, 1998; Beaton e cols., 2000). Assim, para o processo de validação, optou-se pela análise fatorial, que, segundo Pasquali (2001, p. 117), "é, ainda, o melhor método para verificar a hipótese da representação comportamental dos traços latentes em um teste psicológico".

A EMITICE se mostrou um instrumento coeso, enxuto e de fácil compreensão, que mensura o constructo a que se propõe. A escala é fácil de ser aplicada e seus 20 itens podem ser respondidos em poucos minutos. As análises realizadas neste estudo indicaram que a escala possui qualidades psicométricas adequadas, confirmando os cinco fatores que também foram encontrados na literatura.

A desmotivação, descrita na literatura como falta de motivação ou vontade, é o estado de falta de uma intenção de agir. Quando desmotivado, o comportamento da pessoa carece de intencionalidade (Ryan \& Deci, 2000). Esse fator está bem caracterizado na escala proposta, considerando que obteve $\alpha=0,76$. Já a regulação externa, que é o comportamento regulado por meios externos como recompensas, não ficou bem definida na escala, visto ser o fator que apresentou fragilidades, com um item excluído e outro com baixa carga fatorial. $\mathrm{O}$ controle interno ou regulação interiorizada é caracterizado quando uma fonte de motivação externa é internalizada e pode ser exemplificada por comportamentos de culpa e vergonha ou pela necessidade de ser aceito ou ainda pela busca de autoestima (Vansteenkiste e cols., 2006). Esse fator obteve $\alpha=0,71$. O fator controle por identificação, ou regulação identificada, segundo a teoria, ocorre quando se decide tomar determinadas ações devido a situações contingenciais, ou seja, quando não é disponível a escolha. É a forma mais autodeterminada de regulação externa e ocorre quando o indivíduo internalizou os regulamentos externos integrando-os completamente com seus valores pessoais, gerando motivação e comportamentos autodeterminados. Esse fator está bem caracterizado e apresentou $\alpha=0,81$. Por fim, a motivação intrínseca, que se refere a tudo que já foi internalizado e integrado com os demais valores da pessoa, gerando um comportamento intencional a partir das próprias necessidades e vontades do indivíduo, obteve uma boa consistência interna com alpha de 0,87 . Assim, caracterizando comportamentos aos quais já não são necessários reguladores externos para o desenvolvimento da ação (Ryan \& Deci, 2000).

A fidedignidade é um conceito importante e pode ser conhecida como precisão, confiabilidade, consistência interna e estabilidade do instrumento. Como foi visto, o instrumento estudado apresentou alta consistência interna para a escala como um todo e em específico para os fatores de Desmotivação, Controle Interno, Controle por Identificação e Motivação Intrínseca.
Considerando a proposta teórica inicial da Escala (Karsenti, 2008), os resultados deste estudo mostram que a escala pode ser utilizada, embora estudos futuros devam verificar o que pode ter levado à baixa consistência interna do fator de Controle Externo. Além de ter sido necessário retirar o item 2, o item 7 "Porque não existem outras formas de sair-se bem em certos cursos na Universidade" foi o que apresentou menor carga fatorial, levando-se a questionar o fator Controle Externo, que apresenta apenas dois itens com cargas fatoriais altas e positivas.

Apesar das limitações apresentadas, como a baixa consistência interna do fator Controle Externo e a falta de mais informações detalhadas sobre a validação da escala na língua francesa, os resultados demonstram que a Escala EMITICE vem ao encontro da necessidade brasileira de dispor de instrumentos mais compreensivos e abrangentes, que possam ser válidos e úteis em pesquisas e na prática. Destaca-se também por ser um instrumento embasado na Teoria da Autodeterminação, que foi elaborado para avaliar os tipos de motivação intrínseca e extrínseca e que pode colaborar para o melhor entendimento desse conceito na realidade brasileira. As diversas técnicas empregadas demonstraram e reafirmaram a validade e fidedignidade da Escala EMITICE e a viabilidade de sua utilização a partir dos parâmetros psicométricos definidos e investigados.

\section{Considerações finais}

Embora a amostra tenha sido ampla, o desvio padrão não é alto em relação à idade, mostrando uma amostra homogênea e representativa do público estudado, já que todos os pesquisados eram estudantes universitários de cursos na modalidade a distância, no entanto, os dados desta pesquisa não nos permitem universalizar os resultados e conclusões para a população brasileira. Dessa forma, sugere-se que a EMITICE seja aplicada em outras amostras que englobem outras variáveis sociodemográficas, tais como escolaridade, sexo, faixa etária, nível socioeconômico, entre outras, para que se possa identificar de forma mais clara como se manifesta a motivação para as TIC em diferentes grupos e para se obter parâmetros comparativos e se chegar a uma padronização para a população brasileira. Assim, novos estudos podem contribuir nessa direção.

\section{Referências}

Anastasi, A., \& Urbina, S. (2003). Testagem Psicológica. Porto Alegre: Artmed.

Batista-Foguet, J. M., \& Coenders, G. (2000). Modelos de Ecuaciones Estructurales. Madrid: La Muralla.

Beaton, D. E., Bombardier, C., Guillemin F., \& Ferraz, M. B. (2000). Guidelines for the Process of Cross-Cultural Adaptation of SelfReport Measures. SPINE, 25(24), 3186-3191. 
Byrne, B. M. (2010). Structural Equation Modeling with AMOS Basic concepts, Applications and Programming (2nd ed.). New York: Routledge.

Geisinger, K. F. (1998). Psychometric issues in test interpretation. Em J. H. Sandoval e cols.), Test interpretation and diversity: achieving equity in assessment. Washington: APA.

Guimarães, S. E., \& Boruchovitch, E. (2004). O Estilo Motivacional do Professor e a Motivação Intrínseca dos Estudantes: Uma Perspectiva da Teoria da Autodeterminação. Psicologia: Reflexão e Crítica, 17(2), 143-150.

Hair, J. F., Anderson, R. E., Tatham, R. L., \& Black, W. C. (2005). Análise Multivariada de dados. Porto Alegre: Bookman.

Karsenti, T. (2008). Impacto das TIC (Tecnologias de Informação e Comunicação) sobre a atitude, a motivação e a mudança nas práticas pedagógicas dos futuros professores. Em Maurice Tardif \& Claude Lessard (Orgs.), O ofício de professor: história, perspectivas e desafios internacionais (pp. 181-199). Petrópolis, RJ: Vozes.

Noronha, A. P. P., Freitas, F. A., Sartori, F. A., \& Ottati, F. (2002). Informações contidas nos manuais de testes de personalidade. Psicologia em Estudo, 7(1), 1-15. Recuperado: 02 Apr. 2012. Disponível: http://www.scielo.br/pdf/pe/v7n1/v7n1a15.pdf

Pasquali, L. (2001). Técnicas de Exame Psicológico. São Paulo: Casa do Psicólogo/Conselho Federal de Psicologia.
Primi, R., Muniz, M., \& Nunes, C. (2009). Definições comtemporâneas de validade de testes psicológicos. In: Claudio Simon Hutz (Org), Avanços e polêmicas em avaliação psicológica (pp.71-86). São Paulo: Casa do Psicólogo.

Resolução $n^{\circ}$ 02/2003. (2003). Resolução para regulamentação dos testes psicológicos. Brasília, DF: Conselho Federal de Psicologia.

Resolução $n^{\circ}$ 25/2001. (2001). Define teste psicológico como método de avaliação privativo do psicólogo e regulamenta sua elaboração, comercialização e uso. Brasília, DF: Conselho Fedral de Psicologia.

Ryan, R. M., \& Deci, E. L. (2000). Intrinsic and Extrinsic Motivations: Classic Definitions and New Directions. Contemporary Educational Psychology, 25, 54-67.

Vallerand, R. J., Blais, M., Brière, N., \& Pelletier, L. (1989). Construction et validation de l'échelle de motivation en éducation. Revue canadienne des sciences du comportement, 21, 323-349.

Vansteenkiste, M.; Lens, W., \& Deci, E. L. (2006). Intrinsic Versus Extrinsic Goal Contents in Self-Determination Theory: Another Look at the Quality of Academic Motivation. Educational Psychologist, 41(1), 19-31.

Williams G. C, Gagné M., Ryan R. M., \& Deci, E. L. (2002). Facilitating autonomous motivation for smoking cessation. Health Psychol, 21(1), 40-50. Recuperado: 03 Apr. 2012. Disponível: http://www. ncbi.nlm.nih.gov/pubmed/11846344

\section{Sobre os autores}

Patricia Jantsch Fiuza (pjfiuza@yahoo.com.br)

Universidade Federal do Rio Grande do Sul, Porto Alegre, Brasil - Doutoranda

Endereço: Rua Ramiro Barcelos, 2006 - Instituto de Psicologia da UFRGS, sala 122 - Bairro Santa Cecília CEP $90035-003$ - Porto Alegre - RS - Brasil

Jorge Castellá Sarriera (jorgesarriera@gmail.com)

Universidade Federal do Rio Grande do Sul, Porto Alegre, Brasil - Doutor

Endereço: Rua Ramiro Barcelos, 2006 - Instituto de Psicologia da UFRGS, sala 122 - Bairro Santa Cecília CEP 90035-003 - Porto Alegre - RS - Brasil

Lívia Maria Bedin (liviabedin@uol.com.br)

Universidade Federal do Rio Grande do Sul, Porto Alegre, Brasil- Doutoranda

Endereço: Rua Ramiro Barcelos, 2006 - Instituto de Psicologia da UFRGS, sala 122 - Bairro Santa Cecília CEP $90035-003$ - Porto Alegre - RS - Brasil

Relato de pesquisa derivado de tese de doutorado da primeira autora com orientação do segundo autor e colaboração da terceira autora por meio do edital UFRGS EAD 11. 
